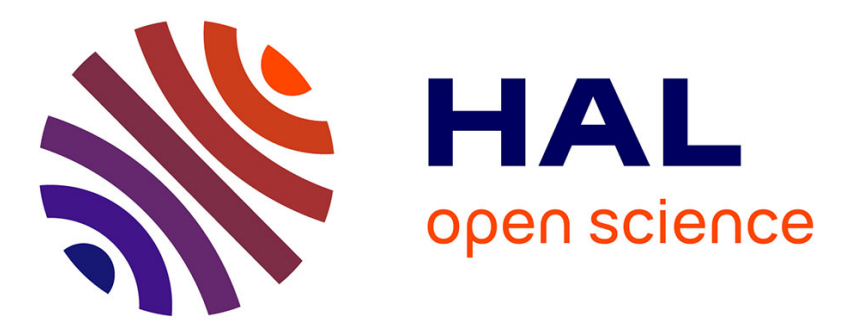

\title{
Shear strength and microstructure of polydisperse packings: The effect of size span and shape of particle size distribution
}

\author{
Emilien Azéma, Sandra Linero, Nicolas Estrada, Arcesio Lizcano
}

\section{- To cite this version:}

Emilien Azéma, Sandra Linero, Nicolas Estrada, Arcesio Lizcano. Shear strength and microstructure of polydisperse packings: The effect of size span and shape of particle size distribution. Physical Review E , 2017, 96 (2), pp.022902. 10.1103/PhysRevE.96.022902 · hal-01576976

\author{
HAL Id: hal-01576976 \\ https://hal.science/hal-01576976
}

Submitted on 24 Aug 2017

HAL is a multi-disciplinary open access archive for the deposit and dissemination of scientific research documents, whether they are published or not. The documents may come from teaching and research institutions in France or abroad, or from public or private research centers.
L'archive ouverte pluridisciplinaire HAL, est destinée au dépôt et à la diffusion de documents scientifiques de niveau recherche, publiés ou non, émanant des établissements d'enseignement et de recherche français ou étrangers, des laboratoires publics ou privés. 


\title{
Shear strength and microstructure of polydisperse packings: The effect of size span and shape of particle size distribution
}

\author{
Emilien Azéma, ${ }^{1, *}$ Sandra Linero, ${ }^{2,3, \dagger}$ Nicolas Estrada, ${ }^{4, \ddagger}$ and Arcesio Lizcano ${ }^{5,8}$ \\ ${ }^{1}$ Laboratoire de Mécanique et Génie Civil (LMGC), Université de Montpellier, CNRS, Montpellier, France \\ ${ }^{2}$ University of Newcastle, Faculty of Engineering and Build Environment, University Dr Callaghan NSW2308, Australia \\ ${ }^{3}$ SRK Consulting (Australasia) Pty Ltd, 10 Richardson St WA6005, Australia \\ ${ }^{4}$ Departamento de Ingeniería Civil y Ambiental, Universidad de Los Andes, Bogotá, Colombia \\ ${ }^{5}$ SRK Consulting (Canada) Inc, 1066 West Hastings St, BC V6E 3X2, Canada
}

(Received 18 April 2017; revised manuscript received 5 July 2017; published 14 August 2017)

\begin{abstract}
By means of extensive contact dynamics simulations, we analyzed the effect of particle size distribution (PSD) on the strength and microstructure of sheared granular materials composed of frictional disks. The PSDs are built by means of a normalized $\beta$ function, which allows the systematic investigation of the effects of both, the size span (from almost monodisperse to highly polydisperse) and the shape of the PSD (from linear to pronouncedly curved). We show that the shear strength is independent of the size span, which substantiates previous results obtained for uniform distributions by packing fraction. Notably, the shear strength is also independent of the shape of the PSD, as shown previously for systems composed of frictionless disks. In contrast, the packing fraction increases with the size span, but decreases with more pronounced PSD curvature. At the microscale, we analyzed the connectivity and anisotropies of the contacts and forces networks. We show that the invariance of the shear strength with the PSD is due to a compensation mechanism which involves both geometrical sources of anisotropy. In particular, contact orientation anisotropy decreases with the size span and increases with PSD curvature, while the branch length anisotropy behaves inversely.
\end{abstract}

DOI: 10.1103/PhysRevE.96.022902

\section{INTRODUCTION}

Whether by physical experimentation or numerical simulation, current research on granular media focuses on materials of increasing complexity. The shape of the particles [1-17], presence of water in the pore space (from capillarity to saturation) [18-21], solid cohesion between particles [22-30], heat transfer [31-35], and fragmentation [36-40] are commonly investigated. Nevertheless, a fundamental characteristic often ignored in research is the system's size polydispersity (i.e., being composed of grains of different sizes).

Polydispersity can result from different natural processes by means of which grains are formed (e.g., fragmentation or mineral precipitation). In addition, in different industries, polydispersity constitutes a target in processes in which grains are produced, transformed, or used. For example, the particle size distribution (PSD) of rock aggregates in concrete is carefully proportioned, with the aim of optimizing the concrete's workability and final strength $[41,42]$. In physics, polydispersity has a strong influence on important properties such as space filling, order-disorder transitions, and fractal structure [43-48].

The role of polydispersity in the microstructure of granular materials has been a subject of interest to the granular physics community, specially focusing on the effects of polydispersity on packing fraction [44,47-51]. Recently, by considering PSDs described by a cumulative $\beta$ function, Voivret and colleagues [44] showed that the densest packing is obtained when the PSD is a uniform distribution by packing fraction.

\footnotetext{
*emilien.azema@umontpellier.fr

†slinero@srk.com.au

${ }^{\ddagger}$ n.estrada22@uniandes.edu.co

§alizcano@srk.com
}

Then, by considering PSDs described by a power law, Estrada [48] showed that the densest packing is obtained for PSDs with an exponent close to 0.5 , which aligns with the experimental work of Fuller and Thompson at the beginning of the twentieth century $[52,53]$.

Besides packing fraction, several researchers have recently investigated the role of polydispersity on shear strength properties. By means of contact dynamics simulations, it was shown that steady-state shear strength is independent of the PSD size span [48,51,54]. To our knowledge, the effects of the shape of the PSD have not yet been the subject of systematic investigation [55], except for the case of frictionless disks with power law PSDs [48].

Thus a better understanding of the complex behavior of granular materials requires characterising the effects that both size span and shape of the PSD have on the mechanical behavior. From a numerical point of view, a major difficulty is the large number of particles required to compose a statistically representative sample and the consequent computational cost. Furthermore, in order to correctly solve the interaction between the larger and the smaller grains, time discretization must be particularly fine.

The aim of this paper is to present a systematic study of the effects of the size span and the shape of the PSD on the shear strength and microstructure of granular samples composed of rigid frictional disks. The simulations were conducted by means of the contact dynamics method [56-58], which is able to cope with stiff frictional contact laws. This method is particularly well suited to the study of dense granular samples with a large number of particles, because the simulations can be performed using large time steps, compared to molecular dynamics simulation approach.

We implemented Voivret's procedure [44] for the systematic numerical generation of collections of particles with a variety of gradings, and analyzed the influence on the shear 
strength of not only the size span but also the shape of the grading curve.

The procedure for the generation of the collection of particles utilizes a normalized $\beta$ function and allows for building gradations similar to those that typically occur in soils [59,60]. Several test samples were numerically built varying the size span of the PSD (from almost monodisperse to highly polydisperse) and the shape of the PSD (from linear to strongly curved). All the collections were analyzed in the steady state in terms of their shear strength, packing fraction, connectivity, and fabric and force anisotropies as functions of the two parameters that control the PSD.

We were able to reproduce previous findings [51,54] showing that the shear strength remains constant across all collections with uniform distributions by packing fraction, irrespectively of their size span. We expand the previous finding showing that the shear strength remains constant for collections that have different shape of the particle size distribution as already suggested by Estrada for frictionless systems [48]. Thus our results generalize Voivret's findings showing that the shear strength is independent of both the size span and the shape of the PSD. From the microstructural point of view, the invariance of the shear strength is underlain by a subtle compensation mechanism that involves both geometrical anisotropies, i.e., contact orientation and branch length anisotropies. This mechanism was already observed previously for uniform PSDs by packing fraction [51,54] and here it is generalized to another group of shapes of the PSD.

In Sec. II, we introduce the numerical approach, system characteristics, and loading parameters. In Sec. III, we focus on the dependence of the shear strength and packing fraction on the PSD parameters. The microstructure is analyzed in Sec. IV in terms of connectivity, and contact and force anisotropies. Section V presents a summary of our main results and some concluding remarks.

\section{MODEL DESCRIPTION}

\section{A. Modeling particle size distribution}

For a systematic investigation of the effect of the size span and the shape of the PSD, careful procedures were implemented in order to generate packings with progressively varying texture. The size span $s$ of the PSD was defined as

$$
s=\frac{d_{\max }-d_{\min }}{d_{\max }+d_{\min }},
$$

where $d_{\max }$ and $d_{\min }$ are the maximum and minimum particle diameters, respectively; $s=0$ corresponds to a monodisperse packing, whereas $s=1$ corresponds to an infinitely polydisperse packing. The size span is related to the size ratio $\lambda$ by the following expression: $\lambda=(1+s) /(1-s)$. The size span was varied in the following range: $s \in[0.2,0.4,0.6,0.8,0.9,0.95]$.

A normalized $\beta$ function was used to model the shape of the PSD. This function was expressed as

$$
\beta(x)=\frac{1}{B(a, b)} \int_{0}^{x} t^{a-1}(1-t)^{b-1} d t,
$$

where, $a>0$ and $b>0$ are the parameters of the distribution and $B(a, b)=\Gamma(a) \Gamma(b) / \Gamma(a+b)$, where $\Gamma$ is the Gamma function. The PSD was then described by means of the
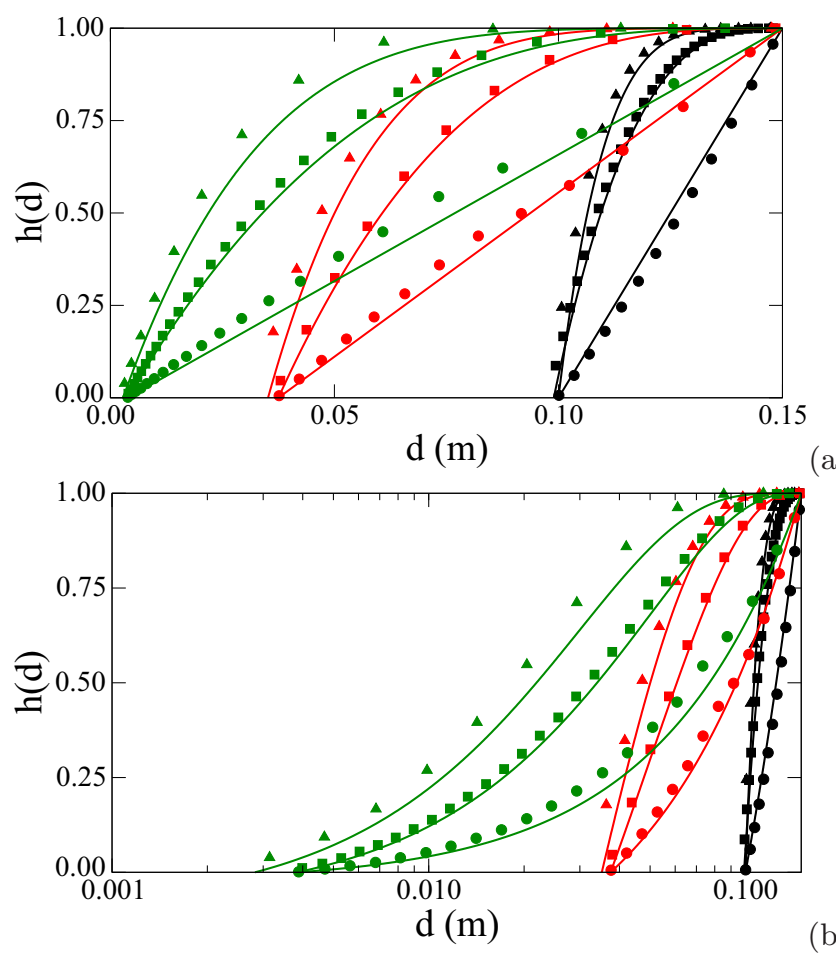

(b)

FIG. 1. Theoretical (solid lines) and generated (symbols) particle size distributions for different combinations of parameters $s$ and $b$. $s=0.2$ (black), 0.6 (red), and 0.95 (green); and $b=1$ (circles), 3 (squares), and 5 (triangles). The same distributions are shown in linear (a) and log-linear (b) scale.

cumulate distribution function $h(d)$, also termed the grading curve, given by

$$
h(d)=\beta\left[x=d_{r}(d) ; a, b\right],
$$

where $d_{r}(d)=\left(d-d_{\min }\right) /\left(d_{\max }-d_{\min }\right)$ is the reduced diameter. As shown by Voivret and colleagues [44], this model allows for controlling both the size span and the shape of the PSD, including reference distributions such as the monodisperse, the power law, and the S-shaped distributions.

In order to generate a set of particle diameters following a given distribution, the following procedure was implemented. First, the range $d_{\max }-d_{\min }$ was divided into $N_{c}$ classes, and the packing fraction in each class was calculated from the theoretical PSD. Then, the set of particles within each class was generated by considering a uniform distribution by packing fraction. In the simulations presented in this paper, $a$ was fixed at 1 and $b$ varied from 1 (a uniform distribution by packing fraction) to 5 (pronounced curvature and $\mathrm{S}$-shaped gradation curve at large values of $s$ in the log-linear representation). In order to ensure statistical representation, a minimum number of particles per class was used. Thus the total number of particles $N_{p}$ increased with the PSD curvature $b$. In practice, $N_{c}$ and $N_{p}$ were chosen in order to: (i) obtain a good fit with the theoretical curve and (ii) obtain a reasonable sample size, in terms of $N_{p} . N_{p}$ increased from 10000 for $b=1$ to $\sim 75,000$ for $b=5$. Figure 1 shows the theoretical PSDs [i.e., Eq. (3)] as well as the PSDs of the generated samples for several combinations of $s$ and $b$. 


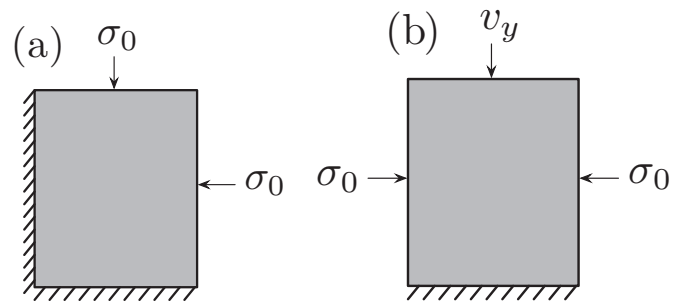

FIG. 2. Boundary conditions for isotropic compression (a) and biaxial shear (b).

\section{B. Packing construction and biaxial test}

The simulations were conducted by means of the contact dynamics (CD) method [56-58], which assumes rigid particles interacting through mutual exclusion and Coulomb friction. The CD method is based on the implicit time integration of the equations of motion and a nonsmooth formulation of mutual exclusion and dry friction between particles. This method requires no elastic repulsive potential and no smoothing of the Coulomb friction law for the determination of forces. The nonsmooth contact laws relate the impulsions exerted at each contact with the changes of relative velocity during the time step. The formulation of these contact laws involves two coefficients of restitution (normal and tangential) that control the amount of energy dissipated during collisions; in all the simulations presented in this paper the coefficients of restitution were set to zero. An iterative algorithm of solution is used by means of which the impulsions and changes of momentum of each grain over the time step are determined. For specific implementation of the CD method, see Ref. [11]. A multipurpose software (i.e., LMGC90) developed at the University of Montpellier was used.

First, for each combination $(s, b)$, a dense packing was built by means of a layer-by-layer deposition strategy [44]. Then, the packings were densified by isotropic compression inside a rectangular frame of dimensions $l_{0} \times h_{0}$, in which the left and bottom walls were fixed and the right and top walls were subjected to the same compressive stress $\sigma_{0}$; see Fig. 2(a). During the isotropic compression phase, the interparticle friction was set to 0.4 . The gravity and the friction coefficient between the particles and the walls were set to zero. This phase was stopped as soon as a persistent contact network was observed and fluctuations of the number of contacts per particle remained below $0.1 \%$ with respect to the mean value. Fragmentation mechanisms were not considered.

Figure 3 shows particle-scale views of the packings obtained for different values of $s$ and $b$ at the end of the isotropic compression phase. Small crystalized regions can be observed for small values of $s$ and large values of $b$, which happens because most of the particles have the same diameter. In contrast, disorder increases with $s$ even for large values of $b$.

The isotropically compressed samples were then subjected to biaxial shear by downward displacement of the top wall, at a constant velocity $v_{y}$ and under constant confining stress $\sigma_{0}$ acting on the lateral walls; see Fig. 2(b). The interparticle and particle-wall friction coefficients were maintained as 0.4 and 0 , respectively. The strain rate $\dot{\varepsilon}$ was low, in order to
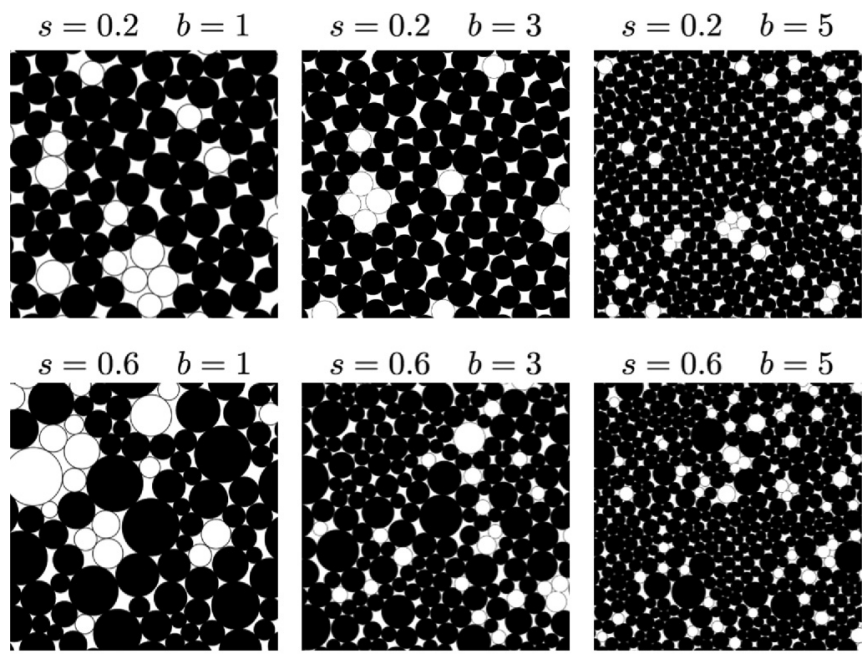

$s=0.6 \quad b=3$

$s=0.6 \quad b=5$
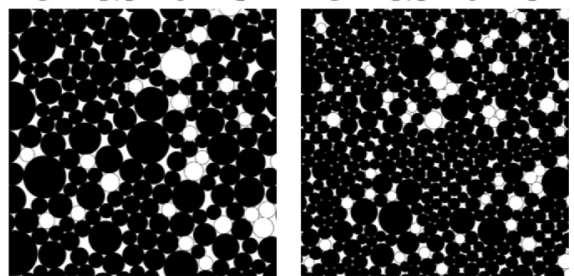

$s=0.95 \quad b=1$
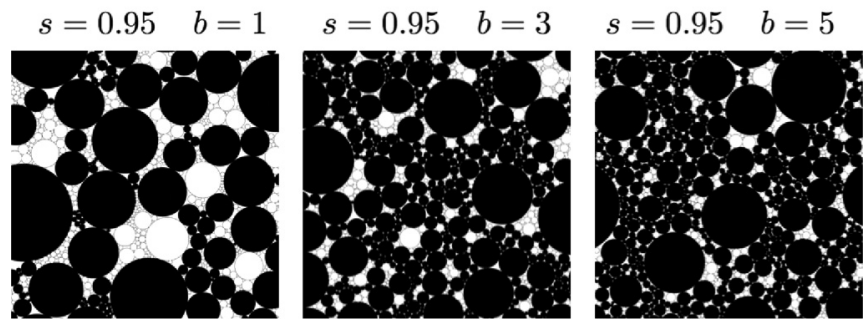

FIG. 3. Particle-scale view at the end of the isotropic compression phase for different combinations $(s, b)$. Floating particles (i.e., particles with one or no contacts) are shown in white.

ensure quasistatic conditions. Quasistaticity conditions were controlled by means of the inertia parameter $I$, given by [61]

$$
I=\dot{\varepsilon} \sqrt{\frac{m}{p}},
$$

where $m$ is the mean particle mass and $p$ is the mean stress. A sheared system can be considered to be at the quasistatic limit if $I \ll 1$. In all simulations presented in this paper, $I$ was below $10^{-3}$. The samples were sheared until a steady state was reached, and the shear strength and the packing fraction were constant. In total, 60 simulations were performed: 30 isotropic compressions and 30 biaxial shear tests.

\section{MACROSCOPIC BEHAVIOR}

\section{A. Definition of macroscopic parameters}

The shear strength of a frictional granular material can be characterized by means of the internal friction angle $\varphi$, which can be calculated from an estimate of the stress tensor $\sigma$. In numerical simulations, $\sigma$ can be evaluated from the contact forces and the geometrical packing configuration. For each particle $i$, one first calculates the internal moment tensor $\boldsymbol{M}^{i}$ as [56]

$$
M_{\alpha \beta}^{i}=\sum_{c \in i} f_{\alpha}^{c} r_{\beta}^{c},
$$

where $f_{\alpha}^{c}$ is the $\alpha$ component of the force exerted at contact $c, r_{\beta}^{c}$ is the $\beta$ component of the position vector of the same contact, and the summation runs over all contacts $c$ in particle $i$. Then, it can be shown that the internal moment of a collection 

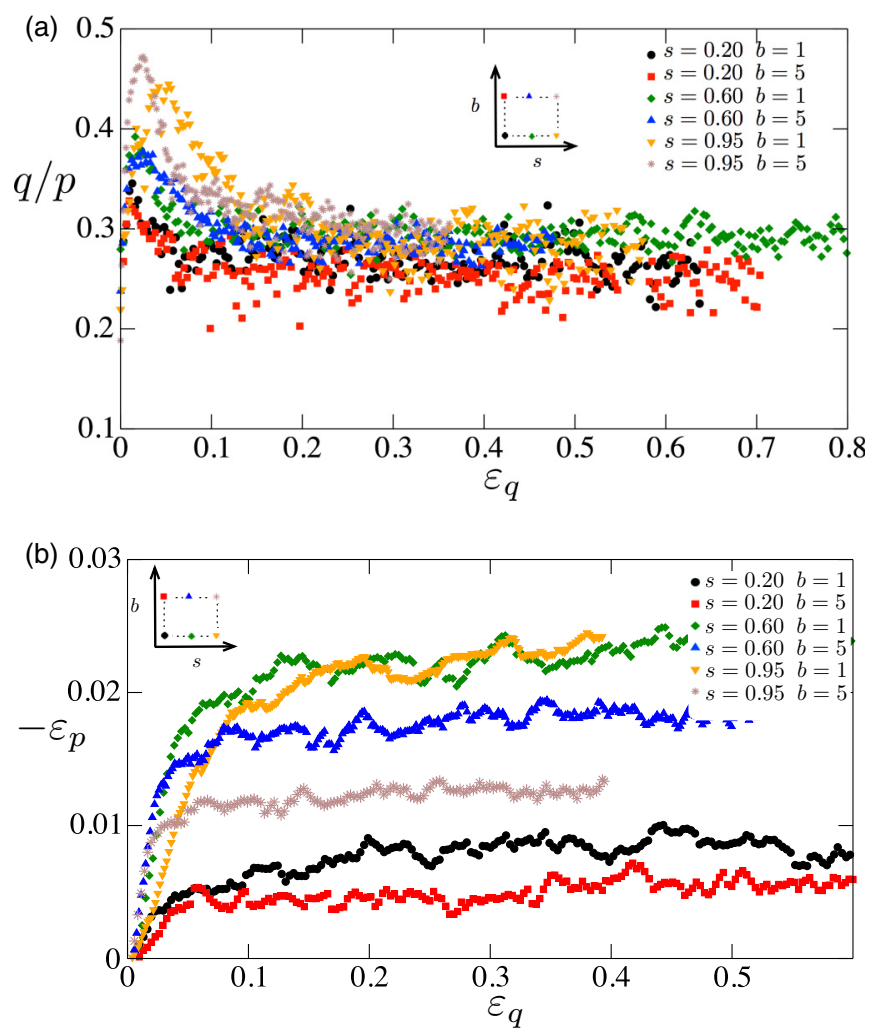

FIG. 4. Stress ratio $q / p$ (a) and volumetric strain $\varepsilon_{p}$ (b) as functions of the deviatoric strain $\varepsilon_{q}$ for different combinations of the size span $s$ and the shape $b$ of the PSD (see schematic representation in the inset).

of particles in a volume $V$ (area in 2D) is the sum of the internal moments of individual particles, and the stress tensor $\sigma$ is simply the density of the internal moment [56]:

$$
\boldsymbol{\sigma}=\frac{1}{V} \sum_{i \in V} \boldsymbol{M}^{i}=\frac{1}{V} \sum_{c \in V} f_{\alpha}^{c} \ell_{\beta}^{c},
$$

where $\ell_{\beta}^{c}$ is the $\beta$ component of the branch vector joining the centers of the contacting particles, and the summation runs over all particles $i$, or contacts $c$, in volume $V$.

The shear strength can be calculated as $\sin \varphi=q / p$, where $q=\left(\sigma_{1}-\sigma_{2}\right) / 2$ and $p=\left(\sigma_{1}+\sigma_{2}\right) / 2$ are the deviatoric and mean stresses, respectively, and $\sigma_{1}$ and $\sigma_{2}$ are the principal stresses. In the biaxial test shown in Fig. 2(b), the major principal stress is oriented along the vertical direction.

The vertical and horizontal strains are given by $\varepsilon_{1}=\Delta h / h_{0}$ and $\varepsilon_{2}=\Delta l / l_{0}$, respectively, where $h$ and $l$ are the height and width of the box, respectively, and $\Delta h=h_{0}-h$ and $\Delta l=$ $l_{0}-l$ are the corresponding cumulative displacements. The deviatoric strain is given by $\varepsilon_{q}=\varepsilon_{1}-\varepsilon_{2}$, and the volumetric strain is given by $\varepsilon_{p}=\varepsilon_{1}+\varepsilon_{2}$.

The packing fraction is the ratio $\rho=V_{p} / V$, where $V_{p}$ is the volume occupied by the particles. The volumetric strain is related to the packing fraction by $\varepsilon_{p}=\Delta \rho / \rho$, with $\Delta \rho=$ $\rho-\rho_{0}$, where $\rho_{0}$ is the initial packing fraction.

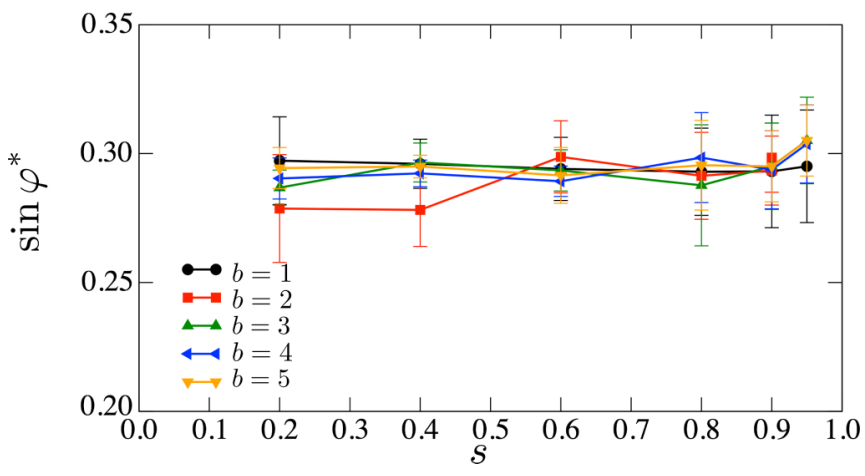

FIG. 5. Sine of the internal friction angle in the steady state $\sin \varphi^{*}$ as a function of $s$ for all values of $b$. Error bars represent the standard deviation in the steady state.

\section{B. Strength and dilatancy}

Figure 4(a) shows the stress ratio $q / p$ as a function of the deviatoric strain $\varepsilon_{q}$ for various combinations $(s, b)$. A classical response is observed, characterized by a stress peak followed by softening and then by a stress plateau. This stress plateau corresponds to the steady state, in which the shear strength fluctuates around a mean value. The rapid increase of $q / p$ at the beginning of the test reflects both the rigidity of the particles and the high initial packing fraction. The volumetric strain $\varepsilon_{p}$ is shown in Fig. 4(b) as a function of $\varepsilon_{q}$ for various combinations $(s, b)$. It is noted that all samples exhibit dilatancy in the initial stage of the test. For $\varepsilon_{q} \geqslant 0.35$, a strain plateau is reached. This strain plateau corresponds to the steady state, characterized by isochoric deformation. Since the samples were sheared at the quasistatic limit and gravity was zero, strain was homogeneously distributed through the sample (i.e., no strain localization was observed). All quantities reported below correspond to the mean values in the steady state, and they are thus independent of the initial configuration of the samples.

Figure 5 shows the sine of the internal friction angle in the steady state $\sin \varphi^{*}$ as a function of $s$ for all values of $b$. It is noted that the shear strength is independent of the size span of the PSD $s$, as shown previously for uniform distributions by volume fraction $[51,54]$. Notably, the shear strength is also independent of the shape of the PSD, controlled by $b$.

Figure 6 shows the packing fraction in the steady state $\rho^{*}$ as a function of $s$ for all values os $b$. It is noted that $\rho^{*}$ increases with $s$, but decreases with $b$. In other words, the systems become denser as the PSD widens, but becomes less dense with increased PSD curvature.

\section{GRANULAR MICROSTRUCTURE}

The system's microstructure can be understood as the quantitative description of the organization of particles, contacts, and forces in space. This organization, which is controlled by steric exclusions and force balance conditions, can be described in terms of various statistical descriptors pertaining to the particles' force-bearing network. This section presents these descriptors as functions of the two parameters controlling the PSD. In addition, the link between some of these descriptors and the system's shear strength is highlighted. 


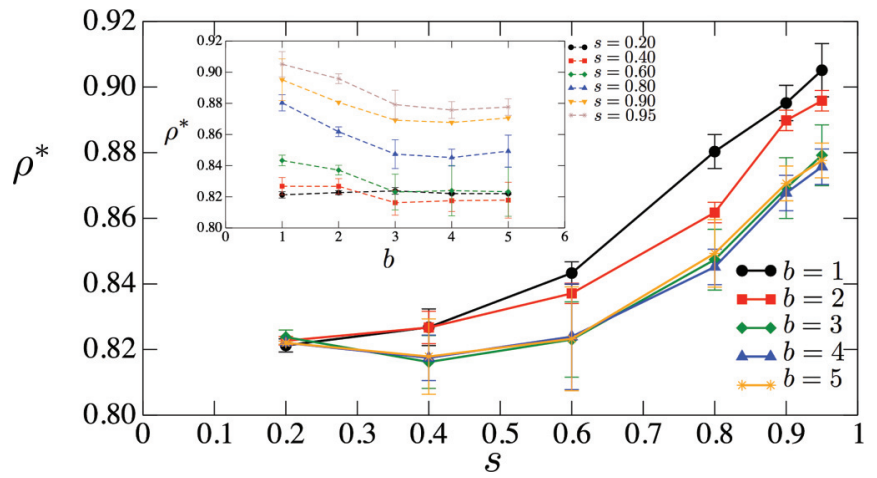

FIG. 6. Packing fraction in the steady state $\rho^{*}$ as a function of $s$ for all values of $b$ (solid lines), and as a function of $b$ for all values of $s$ (inset, dashed lines). Error bars represent the standard deviation in the steady state.

\section{A. Particle connectivity}

Figure 7 shows a snapshot of the system contact network in the steady state, for $s=0.9$ and two extreme values of $b$ (i.e., $b=1$ and 5). Particles connectivity is also shown by means of

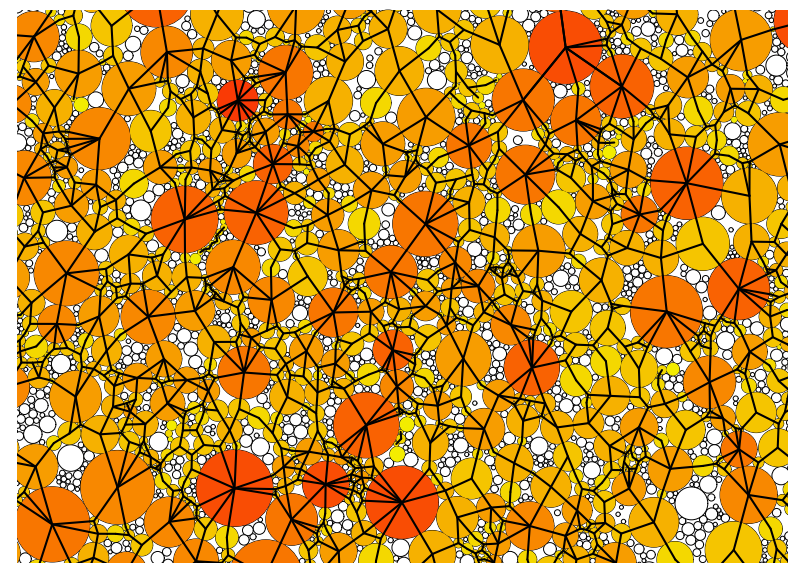

(a)

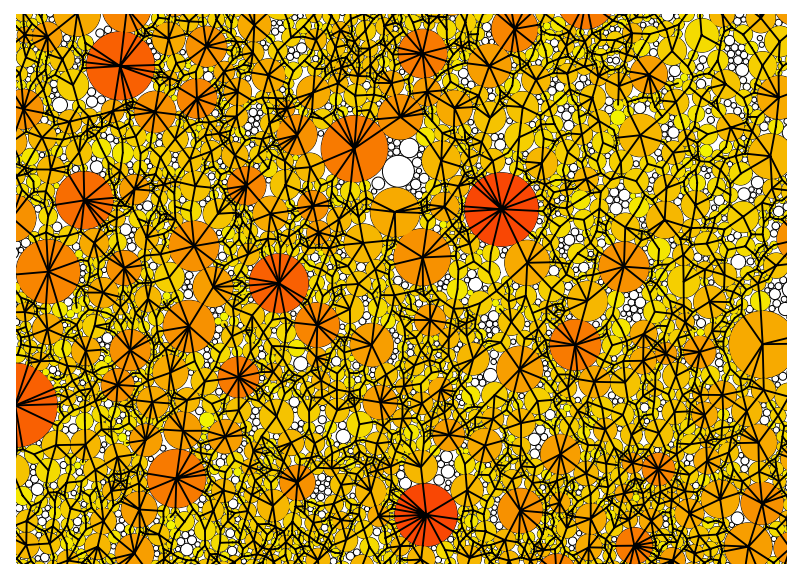

(b)

FIG. 7. Contact network (black lines) and particle connectivity (color scale) for $(s, b)=(0.9,1)$ (a) and $(s, b)=(0.9,5)$ (b). Color intensity is proportional to the grains' number of contacts. The floating particles (i.e., particles with zero or one contact) are shown in white.
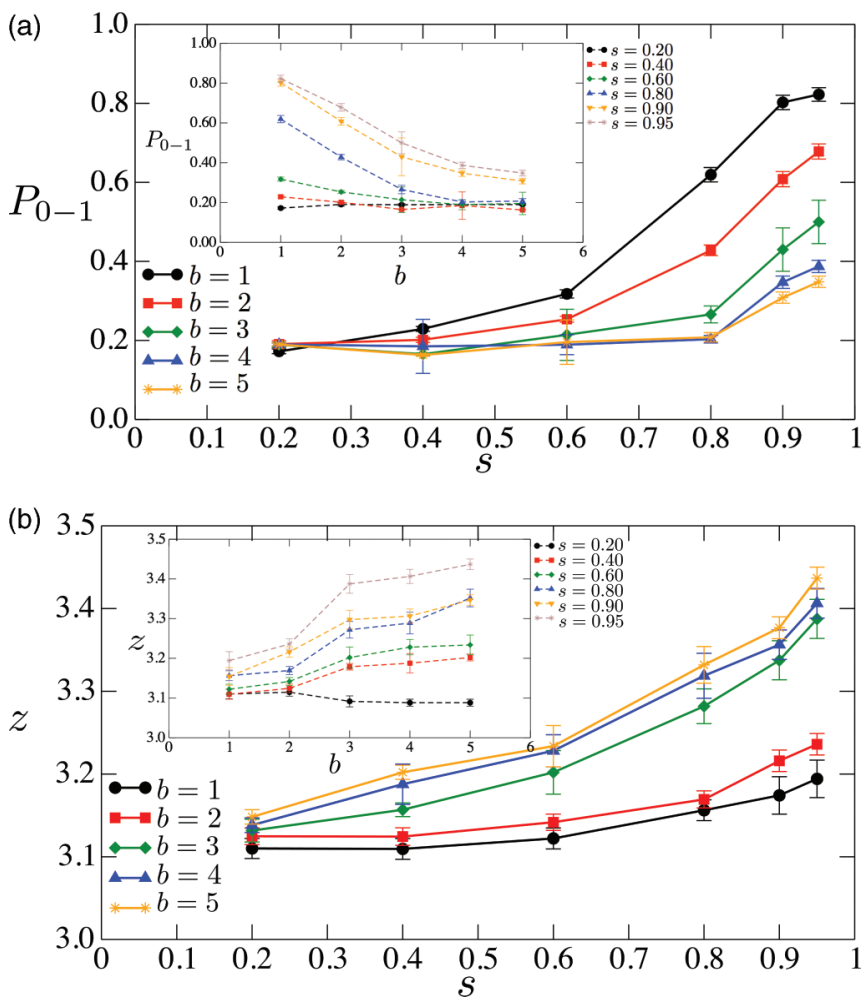

FIG. 8. (a) Proportion $P_{0-1}$ of floating particles and (b) Coordination number $z$, as a functions of $s$ for all values of $b$. The insets show $P_{0-1}$ and $z$ as a function of $b$ for all values of $s$. The error bars represent the standard deviation in the steady state.

a color scale. On the one hand, it can be seen that the largest particles have more contacts than the smaller ones, and the floating particles (i.e., particles with zero or one contact) are predominantly small. Note that, the occurrence of particles with zero contacts is possible due to the fact that gravity is not considered in the simulation. On the other hand, it can be seen that the system with $b=1$ has larger pores than the system with $b=5$, in which the small particles seem to fill the pores better.

The simplest statistical quantities describing the topology of the contact network are the proportion $P_{0-1}$ of floating particles and the coordination number $z$ (i.e., the average number of contacts per particle). Figure 8(a) shows $P_{0-1}$ in the steady state as a function of $s$ for all values of $b$. On the one hand, it can be seen that $P_{0-1}$ increases quickly with $s$. Surprisingly, for the PSD with $b=1$ and $s=0.95, P_{0-1}$ reaches values as high as 0.8 . This means that in systems with a uniform PSD by packing fraction with a large size span, which is the PSD that produces the highest packing fraction, only $20 \%$ of the particles participate in the contact network. On the other hand, $P_{0-1}$ declines towards values between 0.2 and 0.3 as $b$ increases, independently of $s$. The decrease of $P_{0-1}$ with the curvature of the PSD suggests that the smallest particles are increasingly involved in force transmission. Figure 8(b) shows $z$ in the steady state as a function of $s$ for all values of $b$. It is noted that $z$ increases with both $s$ and $b$, from 3.1 to 3.2 for $b=1$ and from 3.15 to 3.45 for $b=5$. In correlation with the variations in $P_{0-1}$, this increase suggests that, in systems where PSDs have high values of $b$, the particles participating 

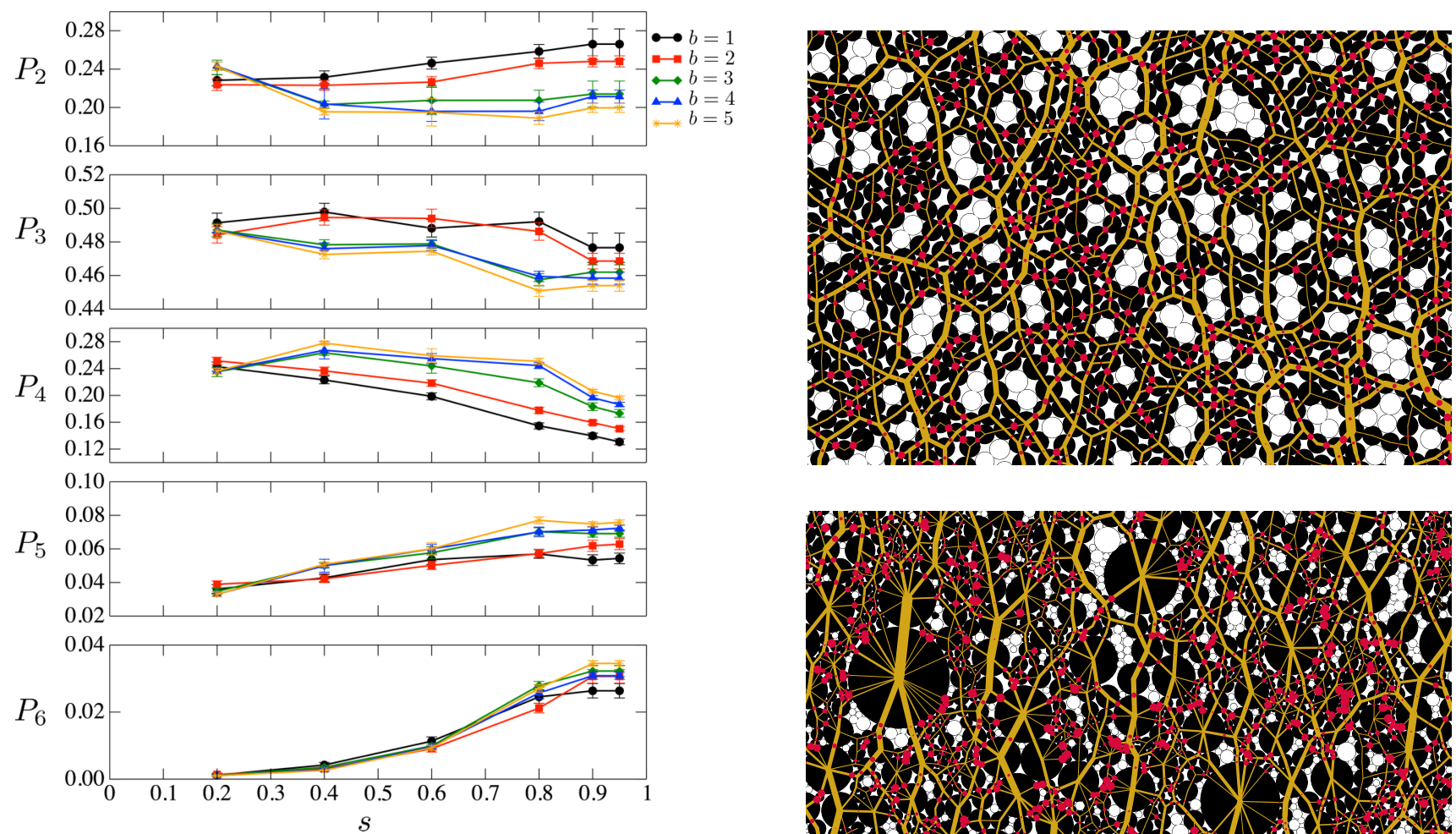

FIG. 9. Particle connectivity, defined as the proportion $P_{c}$ of particles with exactly $c$ contacts, as a function of $s$ for all values of $b$. The error bars represent the standard deviation in the steady state.

in the force network are more connected, even if this network consist of a low proportion of particles.

In order to investigate the contact network further, its connectivity can be characterized by the fraction $P_{c}$ of particles with exactly $c$ contacts. Figure 9 shows $P_{c}$ in the steady state as a function of $s$ for all values of $b$. In the first instance, it is noted that $P_{2}$ is nearly independent of $s$, but decreases with $b$. Secondly, it is also noted that both $P_{3}$ and $P_{4}$ decrease with $s$. However, $P_{3}$ decreases with $b$, while $P_{4}$ increases with $b$. This is strongly correlated with the ordering mechanism explained in Sec. II and shown in Fig. 3. Finally, it is noted that both $P_{5}$ and $P_{6}$ increase with $s$ and $b$. This happens because the relative size of the particles increases with the size span of the PSD, allowing the large particles to be in contact with many small particles. In addition, this effect is enhanced by the curvature of the PSD.

The particles' coordination $z$ is linked to $P_{c}$ by means of the following relationship: $z=\sum_{c=2}^{c=\infty} c P_{c}$. Thus the increase in $z$ with $s$ can be explained by the increases in $5 P_{5}$ and $6 P_{6}$ with $s$. Similarly, the increases in $z$ with $b$ can be explained by the increases in $4 P_{4}, 5 P_{5}$, and $6 P_{6}$ with $b$. The variations of $2 P_{2}$ and $3 P_{3}$ with $s$ and $b$ have a lesser effect on $z$, because of the low value of $c$.

Thus it is notable that the shear strength is independent of the system's connectivity. In other words, a packing with a weakly connected network composed of a large proportion of particles develops the same shear strength that a packing with a well-connected network composed of a low proportion of particles.

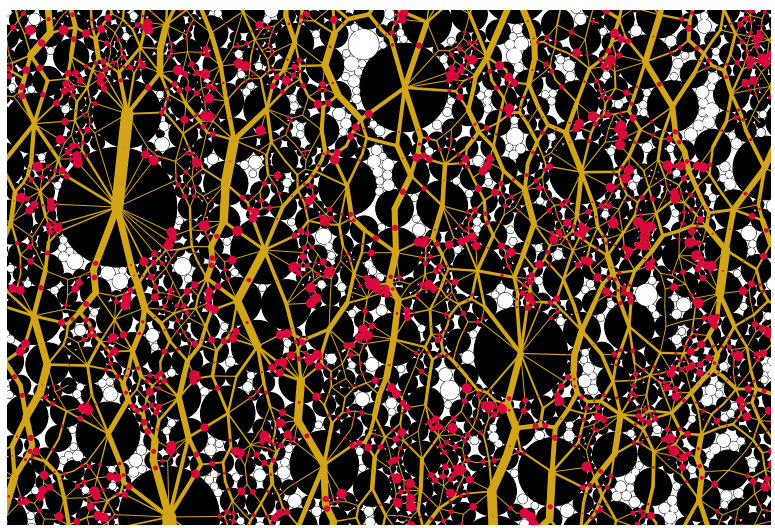

FIG. 10. Snapshots of the force network for (a) $(s, b)=(0.2,2)$ and (b) $(s, b)=(0.9,5)$. Floating particles are shown in white and normal forces are represented by the thickness of the segments joining the particle centers. The red circles in the contact points represent friction mobilization, their diameter being proportional to the friction mobilization index (see text for definition).

\section{B. Anisotropic structures}

Figure 10 shows a typical map of normal forces $f_{n}$ and friction mobilization. The thickness of the lines joining the particle centers is proportional to the magnitude of the normal force. The diameter of the circles drawn at the contact points is proportional to the friction mobilization index $I_{m}=\left|f_{t}\right| /\left(\mu f_{n}\right)$ [62], where $f_{t}$ is the tangential force. It is noted that the force network is anisotropic. It can also be noted, on average, that the stronger forces are oriented along the vertical direction (i.e., that of the major principal stress), whereas small forces are mainly oriented along the horizontal direction [63]. In addition, visual inspection suggests that this bimodal character of the force network is enhanced by polydispersity and that friction mobilization is concentrated in the contacts with the smallest particles.

\section{Harmonic decomposition of stress}

In this section, we briefly recall the stress-force-fabric relationship that allows the material shear strength to be linked to the anisotropic nature of the contact orientations, branch lengths, and force networks $[7,16,64]$. In two dimensions, the unit vector $\boldsymbol{n}$ between two contacting particles can be described 

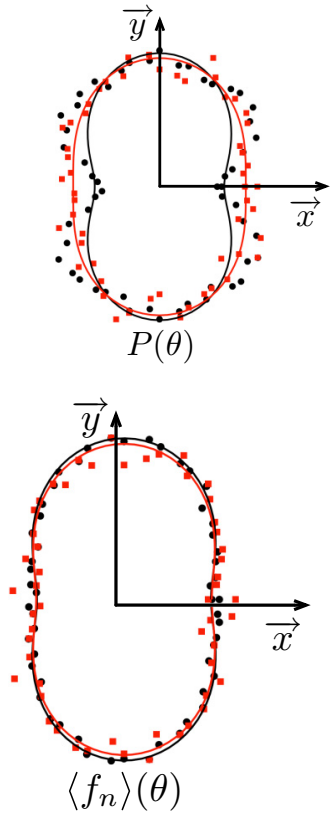

(a)

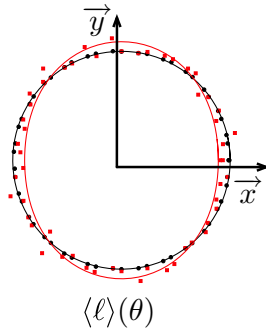

(b)

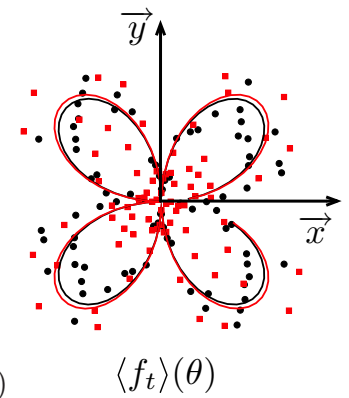

(d)

FIG. 11. Polar representation of the functions $P(\theta)(\mathrm{a}),\left\langle\ell_{n}\right\rangle(\theta)$ (b), $\left\langle f_{n}\right\rangle(\theta)(\mathrm{c})$, and $\left\langle f_{t}\right\rangle(\theta)(\mathrm{d})$, for $(s, b)=(0.2,1)$ (black circles) and $(s, b)=(0.9,1)$ (red squares). The corresponding harmonic approximations are drawn as solid lines.

by an orientation angle $\theta$. The probability density $P(\theta)$ of contact orientations provides useful statistical information about the anisotropy of the contacts network. Along with $P(\theta)$, the anisotropy of the packing can be further characterized by the angular averages of the branch lengths and force magnitudes at the contacts: $\langle\ell\rangle(\theta),\left\langle f_{n}\right\rangle(\theta)$, and $\left\langle f_{t}\right\rangle(\theta)$. These functions describe the general state of anisotropy. In general, as a result of granular disorder, steric exclusions, and mechanical equilibrium, these functions cannot take an arbitrary form. It is usually observed that they can be approximated by the lower-order terms of a Fourier series [7,16,64], as follows:

$$
\left\{\begin{array}{l}
P(\theta)=\frac{1}{2 \pi}\left\{1+a_{c} \cos 2\left(\theta-\theta_{c}\right)\right\} \\
\langle\ell\rangle(\theta)=\langle\ell\rangle\left\{1+a_{\ell} \cos 2\left(\theta-\theta_{\ell}\right)\right\} \\
\left\langle f_{n}\right\rangle(\theta)=\left\langle f_{n}\right\rangle\left\{1+a_{n} \cos 2\left(\theta-\theta_{n}\right)\right\} \\
\left\langle f_{t}\right\rangle(\theta)=\left\langle f_{n}\right\rangle a_{t} \sin 2\left(\theta-\theta_{t}\right)
\end{array},\right.
$$

where $a_{c}$ is the contact orientation anisotropy, $a_{\ell}$ is the branch length anisotropy, $a_{n}$ is the normal force anisotropy, and $a_{t}$ is the friction force anisotropy. The angles $\theta_{c}, \theta_{\ell}, \theta_{n}$, and $\theta_{t}$ are the corresponding privilege directions. The sine function for the expansion of the tangential component $\left\langle f_{t}\right\rangle(\theta)$ is imposed by the requirement that the mean tangential force, given by $\left\langle f_{t}\right\rangle=\int P(\theta)\left\langle f_{t}\right\rangle(\theta) d \theta$, is zero due to the balance of force moments on the particles.

Figure 11 shows a polar representation of the above functions for $(s, b)=(0.2,1)$ and $(s, b)=(0.9,1)$. An anisotropic behavior can be observed for all distributions. The peak values occur along the principal stress direction $\theta_{\sigma}=\pi / 2$ for $P(\theta),\langle\ell\rangle(\theta)$, and $\left\langle f_{n}\right\rangle(\theta)$, whereas the larger values occur at $\theta_{t}=\pi / 4$ for $\left\langle f_{t}\right\rangle(\theta)$. In addition, it can be seen that the angular distributions are indeed well fitted by Eq. (7). In practice, the

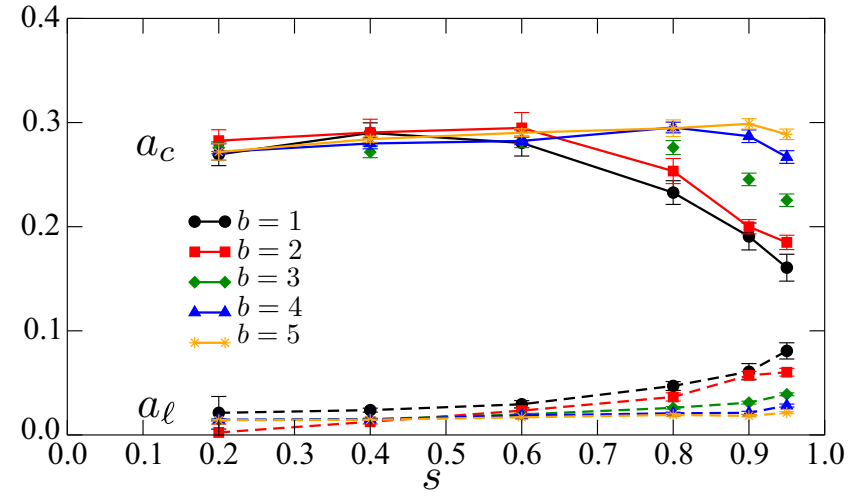

FIG. 12. Contact orientation $a_{c}$ (line) and branch length $a_{\ell}$ (dashed line) anisotropies as functions of $s$ for all values of $b$. Error bars represent the standard deviation in the steady state.

values of all anisotropy parameters can be calculated from generalized fabric tensors as detailed in Refs. [7,16].

The anisotropies $a_{c}, a_{\ell}, a_{n}$, and $a_{t}$ are interesting not only as descriptors of the granular microstructure and force transmission properties, but in that these anisotropies can be linked directly to the internal friction angle through a simple relationship $[7,16,64]$ :

$$
\sin \varphi^{*} \simeq \frac{1}{2}\left(a_{c}+a_{\ell}+a_{n}+a_{t}\right),
$$

where the cross products between the anisotropy parameters have been neglected.

\section{Anisotropies of the contacts and forces networks}

Figure 12 shows the steady-state contact orientation and branch length anisotropies, $a_{c}$ and $a_{\ell}$, respectively, as functions of $s$ for all values of $b$. The same quantities are shown as functions of $b$ for all values of $s$ in Fig. 13. It can be seen that both $a_{c}$ and $a_{\ell}$ are independent of $s$ and $b$ for small values of $s$ (i.e., for $s \leq 0.6$ ). For $s>0.6$, the effect of the shape of the PSD on the evolution of these geometrical anisotropies becomes noticeable. In particular, for low values of $b, a_{c}$ decreases and $a_{\ell}$ increases with $s$. This means that, in systems with PSDs characterized by a low curvature, the increase in the size span of the PSD causes the system to become less anisotropic with regard to contact orientations but at the same time more anisotropic with regard to branch lengths. The former is explained by the increase in connectivity, and the later suggests that strong force chains tend to be "captured" by large particles. In addition, it can be seen that the decrease in contact orientation anisotropy is almost completely compensated by the increase in branch length anisotropy. In the end, geometrical anisotropy is globally independent of $s$, even for large values of $b$.

Figure 14 shows the normal and tangential force anisotropies, $a_{n}$ and $a_{t}$, respectively, in the steady state as functions of $s$ for all values of $b$. Interestingly, both anisotropies are independent of $s$ and $b$. By integrating Eq. (7d) in the range $[0, \pi / 2]$, it can be shown that the mean mobilization index, defined as $\left\langle I_{\mu}\right\rangle=\left\langle\left|f_{t}\right|\right\rangle /\left(\mu\left\langle f_{n}\right\rangle\right)$, is linked to the tangential anisotropy as follows: $\left\langle I_{\mu}\right\rangle=2 a_{t} / \mu$. From Fig. 14, it can be deduced that $\left\langle I_{m}\right\rangle=0.2$. In other words, 
(a)
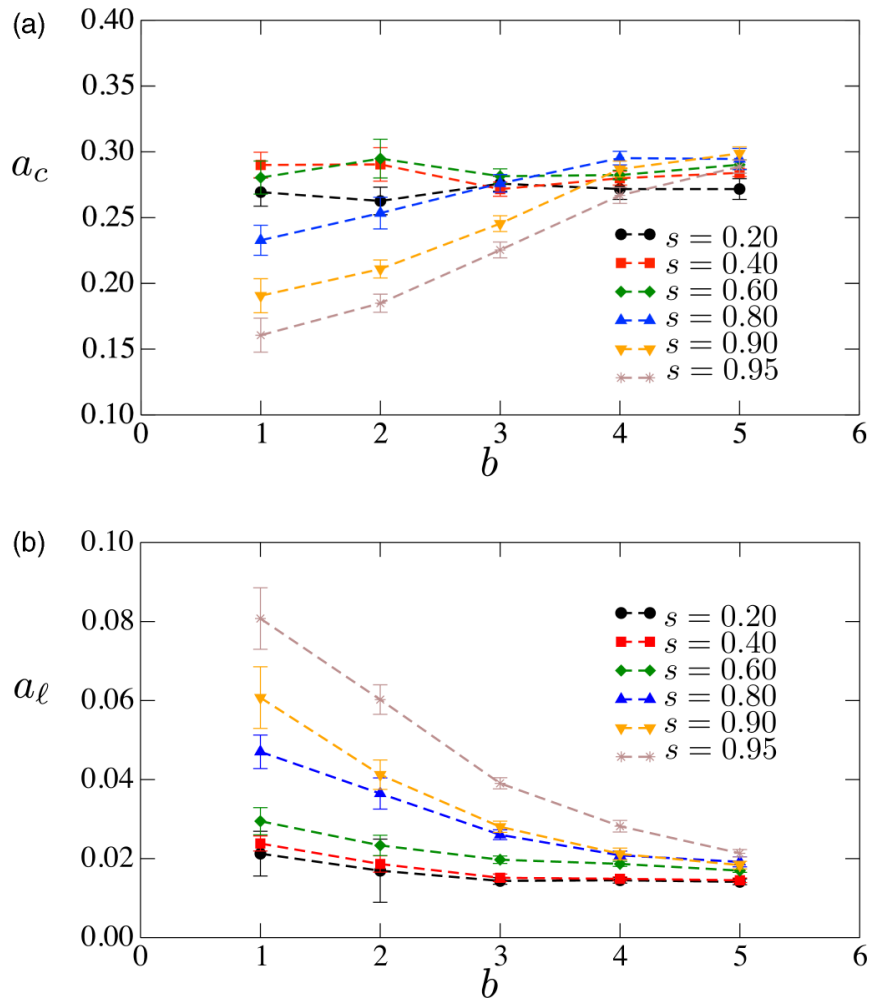

FIG. 13. Contact anisotropies $a_{c}$ (a) and branch length anisotropies $a_{\ell}$ (b) as functions of $b$ for all values of $s$. Error bars represent the standard deviation in the steady state.

even if the location of force chains and friction mobilization is strongly correlated with the size of the grains, as shown in Fig. 10, the above results show that the average force and mobilization values are independent of the PSD.

Thus, based on Eq. (8), it can be seen that the invariance of the macroscopic friction angle in the steady state with the size span and the shape of the PSD results from the opposite variation of $a_{c}$ and $a_{\ell}$ with $s$ and $b$, as well as from the invariance of force anisotropies with the same parameters. Another interesting result here is that the microstructural descriptors of highly polydisperse packings (i.e., $s \geqslant 0.9$ ) with

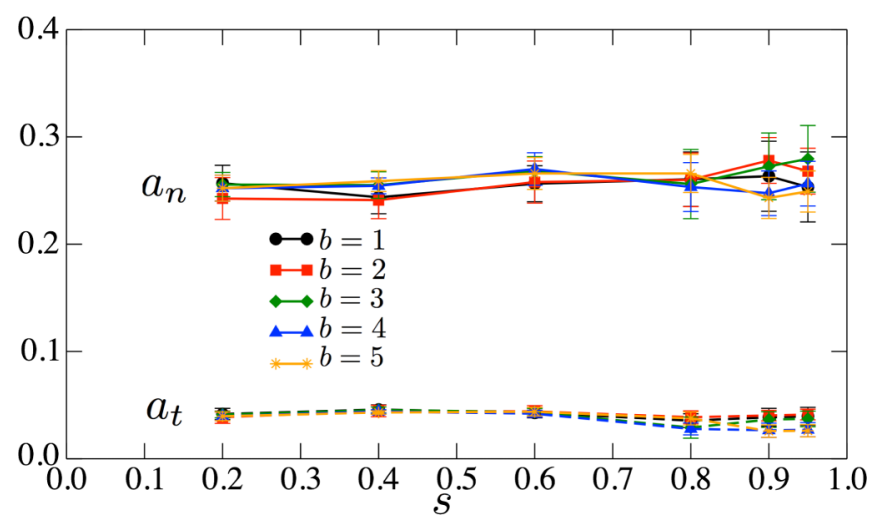

FIG. 14. Normal $a_{n}$ (line) and tangential $a_{t}$ (dashed line) forces anisotropies as functions of $s$ for all values of $b$. Error bars represent the standard deviation in the steady state. a pronounced curvature of the PSD are identical to those of nearly monodisperse packing (i.e., $s \leqslant 0.4$ ) with a linear PSD.

\section{CONCLUSION}

In this paper, an investigation of the combined effects of the size span and the shape of the particle size distribution (PSD) in the rheology of two-dimensional sheared packings is presented. The PSDs were built from a normalized $\beta$ function, according to Voivret's procedure [44], which allowed the size span of the PSD to be varied from nearly monodisperse to highly polydisperse and the shape of the PSD from linear to pronouncedly curved, both in a systematic way. The numerical samples were first isotropically compressed and then sheared in a biaxial configuration until a steady state was reached.

The results of the study confirm previous findings [51,54] indicating that the shear strength is independent of the size span in the case of collections with uniform distributions by packing fraction. In addition, the results show that for a given size span, the shear strength is independent of the shape of the particle size distribution. Thus the methodology allows to generalize Voivret's findings and conclude that the shear strength is independent of both the size span and the shape of the PSD for those shapes that can be described by Eq. (3). The results of the study also show that the solid fraction increases in a nonlinear manner with the size span and decreases with the curvature of the PSD. The shear strength is thus independent of the steady-state solid fraction.

The detailed analysis of the microstructure in terms of the anisotropy of the contact and force networks shows that the independence of shear strength from both size span and shape of the PSD is due to a compensation of anisotropies with geometric origin, as well as due to the invariance of force anisotropies with the PSD. In particular, for PSDs with a low curvature, the decrease of contact orientation anisotropy is counterweighted by the increase in branch length anisotropy. This can be explained by the increase in the proportion of large particles. On the one hand, these particles exhibit large coordination numbers, making the contact network more isotropic. On the other hand, these particles tend to "capture" the force chains, increasing the branch lengths along the principal stress direction. This is not observed in systems with PSDs with a more pronounced curvature, since the proportion of large particles is small and the structure is similar to that of PSDs with low size span, as if the PSD had been "scalped."

The invariance of the shear strength with the PSD is thus something that has been verified by means of numerical simulations with discrete element methods. However, it must be noted that this is different from what is normally observed in experiments, particularly with soils and rock aggregates [65-68]. Our hypothesis is that real granular materials combine several types of polydispersity. In fact, in these materials, several properties such as grain shape and fragmentation susceptibility vary with particle size. In addition, grain-to-grain interaction laws are also size dependent, as cohesive forces become increasingly important compared to gravitational forces as the particle size decreases [69]. The introduction of these other types of polydispersity should help close the gap between numerical simulations and experiments, which would allow numerical simulation to be more widely applied. 
[1] K. Mair, K.-M. Frye, and C. Marone, J. Geophys. Res. 107, EPM 8-1 (2002).

[2] A.-A. Pena, R. García-Rojo, and H.-J. Herrmann, Granular Matter 9, 279 (2007).

[3] S. Sacanna, L. Rossi, A. Wouterse, and A.-P. Philipse, J. Phys.: Condens. Matter 19, 376108 (2007).

[4] S. A. Galindo-Torres, F. Alonso-Marroquín, Y. C. Wang, D. Pedroso, and J. D. M. Castaño, Phys. Rev. E 79, 060301 (2009).

[5] L. Sibille, T. Mullin, and P. Poullain, Europhys. Lett. 86, 44003 (2009).

[6] T.-T. Ng, Mech. Mater. 41, 748 (2009).

[7] E. Azéma and F. Radjaï, Phys. Rev. E 81, 051304 (2010).

[8] R.-C. Hidalgo, I. Zuriguel, D. Maza, and I. Pagonabarraga, J. Stat. Mech. (2010) P06025.

[9] N. Estrada, E. Azéma, F. Radjai, and A. Taboada, Phys. Rev. E 84, 011306 (2011).

[10] CEGEO, B. Saint-Cyr, K. Szarf, C. Voivret, E. Azéma, V. Richefeu, J.-Y. Delenne, G. Combe, C. Nouguier-Lehon, P. Villard et al., Europhys. Lett. 98, 4 (2012).

[11] E. Azéma, N. Estrada, and F. Radjaï, Phys. Rev. E 86, 041301 (2012).

[12] T. Börzsönyi and R. Stannarius, Soft Matter 9, 7401 (2013).

[13] M. Boton, E. Azéma, N. Estrada, F. Radjaï, and A. Lizcano, Phys. Rev. E 87, 032206 (2013).

[14] A. Shimoska, I. Nousou, Y. Shirakawa, and J. Hidaka, Chem. Eng. Trans. 32, 2143 (2013).

[15] E. Azéma, F. Radjaï, B. Saint-Cyr, J.-Y. Delenne, and P. Sornay, Phys. Rev. E 87, 052205 (2013).

[16] E. Azéma, F. Radjai, and F. Dubois, Phys. Rev. E 87, 062203 (2013).

[17] E. Azéma, I. Preechawuttipong, and F. Radjaï, Phys. Rev. E 94, 042901 (2016).

[18] V. Richefeu, M. S. El Youssoufi, and F. Radjai, Phys. Rev. E 73, 051304 (2006).

[19] V. Topin, Y. Monerie, F. Perales, and F. Radjaï, Phys. Rev. Lett. 109, 188001 (2012).

[20] L. Amarsid, in Geomechanics from Micro to Macro, edited by K. Soga (Taylor and Francis Group, London UK, 2015), pp. 467-472.

[21] V. Richefeu, F. Radjai, and J.-Y. Delenne, Comp. Geotech. 80, 353 (2016).

[22] A. Anandarajah, J. Geotech. Eng. 120 (1994).

[23] P. G. Rognon, J.-N. Roux, D. Wolf, M. Naaïm, and F. Chevoir Europhys. Lett. 74, 644 (2006)

[24] A. Taboada, N. Estrada, and F. Radjaï, Phys. Rev. Lett. 97, 098302 (2006).

[25] F. Radjai and V. Richefeu, Philos. Trans. R. Soc. London A 367, 5123 (2009).

[26] N. Estrada, A. Lizcano, and A. Taboada, Phys. Rev. E 82, 011303 (2010).

[27] N. Estrada, A. Lizcano, and A. Taboada, Phys. Rev. E 82, 011304 (2010).

[28] J. Wang, H. S. Yu, P. Langston, and F. Fraige, Granular Matter 13, 1 (2010).

[29] B. Saint-Cyr, F. Radjai, J.-Y. Delenne, and P. Sornay, Phys. Rev. E 87, 052207 (2013).

[30] N. Estrada and A. Taboada, Comp. Geotechnics 49, 62 (2013).
[31] B. Chaudhuri, F.-J. Muzzio, and M.-S. Tomassone, Chem. Eng. Sci. 61, 6348 (2006).

[32] D. Richard, I. Iordanoff, M. Renouf, and Y. Berthier, J. Tribol 130, 031404 (2008).

[33] M. Renouf and N. Fillot, Int. J. Numer. Meth. Engng. 74, 238 (2008).

[34] H. W. Zhang, Q. Zhou, H. L. Xing, and H. Muhlhaus, Powder Technol. 205, 172 (2011).

[35] P. Jongchansitto, X. Balandraud, M. Grédiac, C. Beitone, and I. Preechawuttipong, Soft Matter 10, 8603 (2014).

[36] O. Tsoungui, D. Vallet, and J.-C. Charmet, Powder Technol. 105, 190 (1999).

[37] G. R. McDowell and O. Harireche, Geotechnique 52, 131 (2002).

[38] D. Cantor, N. Estrada, and E. Azéma, Comp. Geotechnics 67, 150 (2015).

[39] D.-H. Nguyen, E. Azéma, P. Sornay, and F. Radjai, Phys. Rev. E 91, 022203 (2015).

[40] D. Cantor, E. Azéma, P. Sornay, and F. Radjai, in Computational Particle Mechanics (Springer, 2016), pp. 1-10.

[41] S. Hentz, F. Donzé, and L. Daudeville, Comp. Structures 82, 2509 (2004).

[42] A. Amirjanov and K. Sobolev, Comp. Concrete 2, 1 (2005).

[43] E. Santiso and E. Muller, Mol. Phys. 100, 2461 (2002).

[44] C. Voivret, F. Radjaï, J.-Y. Delenne, and M. S. El Youssoufi, Phys. Rev. E 76, 021301 (2007).

[45] P. Sollich and N. B. Wilding, Phys. Rev. Lett. 104, 118302 (2010).

[46] U. Agarwal and F. Escobedo, J. Chem. Phys. 137, 024905 (2012).

[47] D.-H. Nguyen, E. Azéma, F. Radjai, and P. Sornay, Phys. Rev. E 90, 012202 (2014).

[48] N. Estrada, Phys. Rev. E 94, 062903 (2016).

[49] T. Aste, Phys. Rev. E 53, 2571 (1996).

[50] R. M. Baram, H.-J. Herrmann, and N. Rivier, Phys. Rev. Lett. 92, 044301 (2004).

[51] D.-H. Nguyen, E. Azéma, P. Sornay, and F. Radjai, Phys. Rev. E 91, 032203 (2015).

[52] W. B. Fuller and S. E. Thompson, Trans. Am. Soc. Civil Eng. 59, 67 (1907).

[53] F. W. Taylor, Critical Evaluations in Business and Management, edited by J. C. Wood and M. C. Wood, 1 ed. (Routledge, 2002).

[54] C. Voivret, F. Radjaï, J.-Y. Delenne, and M. S. El Youssoufi, Phys. Rev. Lett. 102, 178001 (2009).

[55] J. Wiacek and M. Molenda, Int. J. Solids Struct. 51, 4189 (2014).

[56] J.-J. Moreau, Eur. J. Mech. A/Solids 13, 93 (1994).

[57] M. Jean, Comput. Methods Appl. Mech. Eng. 177, 235 (1999).

[58] F. Radjai and V. Richefeu, Mech. Mater. 41, 715 (2009).

[59] J. M. Duncan and S. G. Wright, Soil Strength and Slope Stability, 1 ed (Wiley, 2005).

[60] J. Mitchell and K. Soga, Fundamentals of Soil behavior (Wiley, 2005).

[61] G. D. R. Midi, Eur. Phys. J. E 14, 341 (2004).

[62] E. Azéma and F. Radjaï, Phys. Rev. E 85, 031303 (2012).

[63] F. Radjai, D.-E. Wolf, M. Jean, and J.-J. Moreau, Phys. Rev. Lett. 80, 61 (1998).

[64] L. Rothenburg and R.-J. Bathurst, Geotechniques 39, 601 (1989). 
[65] E. Frossard, W. Hu, and C. Cano, Geotechnique 62, 415 (2012).

[66] J. Lowe, Shear strength of coarse embankment dam materials, Proceedings of the Eighth International Congress on Large Dams (ICOLD, Scotland, Edinburgh, 1964), pp. 745-761.

[67] N. D. Marachi, C. K. Chan, and H. B. Seed, J. Soil Mech. Found. Div., Am. Soc. Civ. Eng. 98, 95 (1972).
[68] J. Zeller and R. Wullimann, in Proceedings of the Fourth International Conference on Soil mechanics and Foundation Engineering ISSMGE, ISSMGE (ISSMGE, London, 1957), Vol. 2, pp. 399-404.

[69] E. J. R. Parteli, J. Schmidt, C. Blümel, K.-E.. Wirth, W. Peukert, and T. Pöschel, Sci. Rep. 4, 6227 (2014). 